\title{
COMERCIO JUSTO: TRADUCCIÓN Y COOPERACIÓN EN ECUADOR
}

\author{
Betty Espinosa \\ bespinosa@flacso.org.ec \\ FACULTAD LATINOAMERICANA DE CIENCIAS SOCIALES, ECUADOR
}

\section{RESUMEN}

Se propone una lectura ${ }^{1}$ de la problemática del comercio justo en tanto constituye un conjunto de acciones de traducción, realizadas por organizaciones que se sitúan como intermediarias entre realidades sociales distintas. En la primera parte presentamos los actores de esta problemática y damos cuenta de los enfoques «clásicos» del comercio justo. Nosotros optamos por seguir a Callon y Latour (1997) para decir que los actores de comercio justo tienden a situarse simultáneamente en los registros del «don» y del «intercambio». Para comprender las profundas diferencias y semejanzas entre estos dos registros así como su dinámica de coexistencia, en la segunda parte presentamos un estudio de caso de una organización no gubernamental en Ecuador, que permite ilustrar los esfuerzos y dificultades del comercio justo en el contexto de la globalización.

Palabras clave: Don, procesos de traducción, artesanías, cacao. 


\begin{abstract}
This essay proposes a reading ${ }^{2}$ of the problem of fair trade in the sense that it constitutes a whole of actions of translation carried out by organizations situated as intermediaries between distinct social realities. In the first part, we present the actors of this situation and we recall the "classic" approaches of fair trade. We opt to follow the precepts of Callon and Latour (1997) to say that the actors of fair trade tend to situate themselves simultaneously in the registries of "gift" and of "exchange." To understand the profound differences and similarities between these two registries as well as their dynamic of coexistence, in the second part we present a case study of a nongovernmental organization in Ecuador that illustrates the efforts and difficulties involved in fair trade in the context of globalization.
\end{abstract}

Key words: Gift, translation processes, crafts, cacao. 


\section{PROBLEMÁTICA}

\section{LOS ACTORES Y SUS RELACIONES}

En todo el mundo, norte y sur, encontramos organizaciones no gubernamentales que actúan como intermediarias entre pequeños productores del sur - a menudo agrupados en cooperativas o grupos informales de producción - y consumidores del norte y del sur, que comparten o no principios de solidaridad con los productores. Es a través de este tipo de organizaciones que el café Max Havelaar, la miel Maya, los productos OXFAM, el cacao que se trasformará luego en chocolates, y numerosos productos artesanales y agrícolas de América Latina, llegan a desplazarse en la misma región así como hacia Europa y Norteamérica.

Este tipo de relación que aboga por el comercio justo aparece a comienzos de los años 60. En el origen se encontraban preocupaciones crecientes en cuanto al lugar de los países en desarrollo en el comercio internacional, especialmente la problemática de los términos de intercambio en condiciones de desigualdad. Estas preocupaciones condujeron a que dichos países propusieran la creación de un dispositivo en Naciones Unidas y dieran lugar a la Conferencia de Naciones Unidas sobre Comercio y Desarrollo, UNCTAD, que se reunió por primera vez en Ginebra en 1964 y que se convoca cada cuatro años. Además se creó el Grupo de los 77 —que actualmente cuenta con 131 miembros— ${ }^{3}$. Paralelamente, la Iglesia católica reaccionó también en 1967 a través de la encíclica Populorum Progressio sobre el desarrollo de los pueblos, que hace un llamado a promover «un verdadero desarrollo que no sea la riqueza egoísta y amada por sí misma, sino la economía al servicio del hombre» ${ }^{4}$. Esta última declaración tuvo un impacto particularmente importante en 
América Latina y se encuentra en la base de diversos proyectos de implicación de la Iglesia con el sector del desarrollo.

Pero no fue sino con el esfuerzo de organizaciones no gubernamentales que este pensamiento pudo traducirse en acciones concretas. En Ecuador, la primera organización de este tipo fue el Fondo Ecuatoriano Populorum Progressio, FEPP, fundado en 1970. Luego, algunas otras organizaciones sin fines de lucro han intentado por diversos medios generar alternativas de producción, comercialización y servicios financieros para apoyar a pequeños productores agrícolas y de artesanías. Este tipo de organización adopta criterios de definición tales como un precio mínimo o precio justo pagado a los productores, la disminución de la cadena de intermediarios, el respeto de las condiciones mínimas de trabajo según la definición del país o de la Oficina Internacional de Trabajo, OIT, la contribución al prefinanciamiento de la producción y, de ser necesario, la asistencia técnica a la producción. ${ }^{5}$

Estas organizaciones se han reagrupado en el ámbito internacional desde finales de los años 80 en cuatro asociaciones: 1) International Fair Trade Association, IFAT, ${ }^{6}$ que reúne alrededor de trescientas organizaciones de productores de países en desarrollo y las organizaciones que los apoyan en el norte y el sur; 2) Fair Trade Labelling Organizations, FLO, organización internacional de la etiqueta comercio justo que agrupa las iniciativas de etiquetaje provenientes de catorce países europeos, así como de América del Norte y Japón; 3) European Fair Trade Association, EFTA, que agrupa una docena de centrales de compra importadoras en nueve países europeos; 4) Network of European World Shops, NEWS, que reúne las asociaciones nacionales de las Tiendas del Mundo, unas 2.700 en 13 países. 
En 1991, estas cuatro organizaciones se reunieron en la red internacional FINE: FLO, IFAT, NEWS y EFTA. En estos espacios se define el comercio justo como un «asocio comercial fundamentado en el diálogo, la trasparencia y el respeto, cuyo objetivo es lograr una mayor equidad en el comercio mundial. Se contribuye al comercio durable ofreciendo las mejores condiciones comerciales y garantizando los derechos a los productores y a los trabajadores marginalizados, especialmente a aquellos del Sur...» ${ }^{7}$.

Sin embargo, esta forma institucionalizada de apoyo ha sido objeto de críticas por parte de los productores, mismas que fueron expresadas tanto en el Foro Social Mundial -Porto Alegre 2005- ${ }^{8}$, como en el Congreso Mundial de la Asociación Internacional de Comercio Justo IFAT —Quito 2005- Los productores perciben que se han desarrollado nuevas barreras a la entrada de los mercados, puesto que — según ellos — lo que se impone en estos espacios es ante todo las preocupaciones del norte, especialmente la trasparencia de información al consumidor. Se han originado múltiples tensiones alrededor de temas como: la desconfianza suscitada por el crecimiento del movimiento y el peligro de canalización de los conceptos de base, los peligros de una política basada en el comercio exterior, las amenazas de recuperación de los conceptos por parte de grandes grupos privados, las dificultades de medición del impacto de las acciones, las dificultades de la gestión de marcas de comercio justo y, de manera más general, la gran heterogeneidad de las organizaciones miembros. ${ }^{9}$ Asimismo, los productores expresan dudas sobre los procesos de certificación de la calidad y sobre el papel de Fair Trade Labelling Organizations, FLO. 
Estas críticas internas cuestionan los cambios a los cuales las organizaciones de comercio justo se ven confrontadas actualmente en el marco de la globalización de la economía. Estas organizaciones tejen actualmente redes cuyos límites no excluyen las organizaciones convencionales con el objetivo de optar por estrategias de crecimiento y de diversificación. Así han ingresado en las redes convencionales de comercio y trabajan a la vez con grandes y pequeños productores, ya sea en esquemas de cooperación de larga duración o a través de relaciones de corto plazo.

\section{COMPRENSIONES DEL COMERCIO JUSTO A PARTIR DE LA ECONOMÍA SOCIAL Y SOLIDARIA}

Para comprender la problemática planteada es indispensable empezar por situar de manera más precisa el comercio justo y sus organizaciones con respecto a nociones como aquellas de la economía social y de la economía solidaria, dado que estas incluyen las asociaciones, las empresas cooperativas y las mutuales o mutualistas. La economía social se considera como un «tercer sector», distinto de los sectores público y privado. En la literatura especializada, sobre todo en la tradición anglosajona, se las aborda como «organizaciones sin fines de lucro —non profit organizations, NPO—, sector independiente u organizaciones voluntarias» ${ }^{10}$, mientras en América Latina se las denomina con frecuencia como organizaciones no gubernamentales. La mayor parte de teorías, que enfocan ya sea la oferta o la demanda, se sitúan en lo que Favereau (1989) denomina como «teorías estándar ampliadas» $\mathrm{o}$ «teorías contractualistas» ${ }^{11}$, mismas que explican la existencia de este tipo de organización a partir de las fallas del mercado o del Estado. 
Weisbrod (1977) percibe NPO como un conjunto de productores privados de bienes públicos que intervienen cuando una parte de individuos se encuentra insatisfecha de los bienes provistos por el Estado, ya sea en el ámbito cuantitativo o cualitativo. En este sentido se habla de un fracaso del Estado. Fama y Jensen (1983) plantean la teoría del principal y el agente para comprender el problema de las asimetrías de información entre individuos, y consideran que las NPO tienen ventajas sobre las empresas privadas debido a la falta de distribución de utilidades. Williamson (1979) postula que el comprador de un servicio — principal — no puede controlar por diversas razones el desempeño del prestador —agente-, por lo que el primero tendrá interés en establecer contratos que inciten al segundo a actuar en función del principal; en este marco las NPO ofrecerían una forma de control de los riesgos de comportamientos oportunistas. Según Hansmann (1980) no solo la asimetría de información puede generar la proliferación de NPO. La «teoría del control ejercido por el consumidor» explicaría la decisión de los consumidores de optar por la organización de tipo cooperativas o clubes, en los que hay una evaluación previa de la calidad del candidato al ingreso. Recurrir a las NPO puede ser además una manera de asegurar criterios de buen uso de recursos. ${ }^{12}$ En un sentido que privilegia el enfoque de la oferta, Ben-Ner y Van Hoomissen (1991) proponen la teoría de los «stakeholders» al definir las NPO como coaliciones de individuos que se asocian para proveerse ellos mismos, o a otras personas, bienes y servicios que no están siendo adecuadamente entregados por organizaciones con fines de lucro o por el Estado.

Sin embargo, la hipótesis de racionalidad sustancial optimizadora que subyace a las teorías anteriores plantea al menos dos problemas de lectura. Se instaura una especie de 
jerarquía entre organizaciones, mediante la cual aquellas que se pueden designar como de economía social no serían sino una respuesta al fracaso de los arreglos del mercado o del Estado y que, por tanto, podrían «regresar» al ámbito público o del mercado en un momento posterior. Desde este prisma, se les atribuye un rol residual y se construye una visión contrastante al oponerlas a las organizaciones gubernamentales o a aquellas con fines de lucro. ${ }^{13}$ Una segunda consecuencia importante de esta lectura es que se llega a definir las organizaciones únicamente como espacios de contratos entre individuos (Favereau 1989). Esta concepción reduce la realidad social a una suma de individuos que reaccionan a mecanismos incitativos para maximizar su utilidad individual.

Por otra parte, Defourny et al. (1998) recurren a un análisis histórico para dar cuenta de la existencia de la economía social, misma que según estos autores está ligada a la historia de la cuestión social en Europa. En efecto, su línea cooperativa se desarrolló en un inicio entre las clases obreras que luchaban por mejorar sus condiciones de vida muy precarias. A partir de esta constatación se resalta una primera dimensión de la economía social, que es aquella ligada a la condición de «necesidad» por la que se busca responder a las fallas de la economía dominante, en particular a los deseos no cubiertos por el mercado. Una segunda dimensión que explica la movilización social consiste en el deseo de pertenencia a un mismo grupo, a un destino común, por el cual se forja una «identidad colectiva». La economía social nace de movimientos que pretenden construir, a través de actividades económicas, relaciones sociales de solidaridad. Finalmente, refiriéndose a Touraine, se incluye una tercera dimensión, que es la de un «proyecto» que da sentido a esa identidad, a ese querer vivir en comunidad. 
Los rasgos distintivos, en este marco, de la economía social son: a) «finalidades» que apuntan a cumplir funciones sociales y no a servir los intereses de aquellos que detienen el capital, en el sentido de que sus actividades buscan asegurar a la vez la viabilidad económica y la utilidad social; b) «actores y beneficiarios» que pasan por dificultades; c) «estructuras de reglas» orientadas a la participación y que no reparten el poder en función del capital detenido; d) «actividades» de carácter empresarial, en el sentido de que la producción de bienes y servicios se desarrolla para asegurar el autofinanciamiento al mismo tiempo que se busca el apoyo de los poderes públicos. ${ }^{14}$

En lo que concierne a la economía solidaria, según Defourny et al. (1998), representaría una parte de la economía social que asumiendo los límites ligados a una economía de mercado se distingue cualitativamente de ésta por la búsqueda de objetivos sociales y económicos y por un modo de gestión asociativo. Si las empresas lucrativas tienen tendencia a dejar que recaigan sobre los individuos o las colectividades públicas sus funciones de utilidad social, las empresas asociativas las internalizan. La economía solidaria «busca reforzar simultáneamente la pertenencia social a una comunidad y la creación de empleo, cuestionando de esta manera la lógica de adaptación al modo de producción mercantil» 15 .

\section{PUNTO DE PARTIDA PARA UNA COMPRENSIÓN RENOVADA DEL COMERCIO JUSTO}

Si bien las teorías expuestas en el punto anterior explican de manera parcial y muchas veces complementaria algunos rasgos de las organizaciones enmarcadas en la economía social y solidaria, nos interesa examinar el acento en las actividades portadoras de relación social, 
que se logra gracias a una hibridación de recursos humanos — asalariados y voluntarios—, y financieros — subvenciones públicas, cotizaciones y donaciones, venta de servicios— ${ }^{16}$. Asimismo quisiéramos resaltar el carácter de «solidaridades próximas» que se promueven a través de estas organizaciones y que, según R. Castel, ${ }^{17}$ se oponen a las solidaridades más abstractas de las instituciones de economía social que lo que producen es una solidaridad organizada y anónima. De hecho, el comercio justo se autodefine por ser una respuesta ${ }^{18}$ a $^{2}$ los efectos desestructurantes de una economía cada vez más competitiva e individualista. A través de las redes de comercio justo se pretende remunerar mejor el trabajo de los productores del sur, agrupados en cooperativas. En este sentido, nos parece confirmarse la percepción de Salamon (1995), para quien este tipo de organizaciones son pioneras en rescatar las demandas sociales emergentes y, por tal motivo, tienen el privilegio de actuar en contextos nuevos y complejos.

Un rasgo que nos parece particularmente importante es el hecho de que ciertas organizaciones de economía solidaria se constituyen en intermediarios, en traductores de una cadena que va de los pequeños productores a los consumidores. Esto no tendría nada de específico si no fuera porque al hacerlo se permite - en cierta forma- el encuentro de lógicas y prácticas distintas. Por una parte, se encuentran productores que reproducen las características de una economía tradicional, tal como la describe P. Clastres (1980) ${ }^{19}$ : una lógica económica de abundancia ${ }^{20}$ e imbricada que se resiste a separarse de las esferas social y política, fundamentada en el deseo de independencia, en la reciprocidad como privilegio y deber, en un principio antiexcedentes. Del otro lado de la cadena se encuentran consumidores que pueden compartir y pueden querer contribuir a la realización de esas 
finalidades, o simplemente aquellos que compran los productos sobre la base de una evaluación clásica de la relación entre calidad y precio en el marco de un modelo racional de comportamiento.

En la lógica del «intercambio», que es también aquella del cálculo, prevalece la dinámica del mercado o de los mercados ${ }^{21}$ como espacios reales y simbólicos donde se realizan las transacciones. Lo que se encuentra aquí son consumidores y vendedores que encarnan la noción de individuo que toma decisiones racionales. Como lo señalan M. Callon y B. Latour, esos individuos realizan transacciones que se cierran con el pago de un precio y la entrega de la mercadería, sin que sea necesario que se vuelva a producir un intercambio; «entran y salen del intercambio como extraños» ${ }^{22}$. Mientras que en el registro del «don» lo que importa es precisamente lo contrario, no separarse nunca de un proceso de «dar-recibir-devolver» que se mantiene indefinidamente.

Hay que tomar en cuenta además que las relaciones en el mundo del desarrollo, es decir, entre técnicos del desarrollo y los beneficiarios de esa cooperación, están marcadas por la asimetría, en el sentido que N. Zaccaï-Reyners da a este concepto: «los términos que están implicados no pueden ser intercambiados sin que su naturaleza misma sea modificada. Alter no puede tomar el lugar de ego por diversos motivos...» ${ }^{23}$.

En nuestro estudio nos cuestionamos sobre el contenido de las acciones de «traducción» que realizan las organizaciones de comercio justo entre esas lógicas distintas, esto es, sobre las acciones de cambio que realizan entre un enunciado con significado para un grupo de personas hacia otro enunciado con el mismo significado para otro grupo. Nos preocupa precisamente ese proceso de traducción por el que — parafraseando a F. 
Rosenzweig - ${ }^{24}$ se sirve a dos amos, al extranjero en su extranjeridad, al «beneficiario» en su deseo de apropiación de algo que no es.

En la siguiente sección nos proponemos identificar la conflictiva relación entre el «don»y el «intercambio», fundamentalmente a partir de las relaciones entre «don»e «intercambio» identificadas por M. Callon y B. Latour (1997). Analizamos la historia de una organización no gubernamental ecuatoriana dedicada al comercio justo desde comienzos de los años 80, a la que nos referiremos en este artículo como «ONG». Este caso nos permitirá conocer los esfuerzos y dificultades que tienen actualmente las organizaciones de comercio justo en el marco de la globalización y de las nuevas exigencias del mercado, percibir cómo estas organizaciones cambian y se resisten al cambio, así como los encuentros y desencuentros que se producen. La investigación se realizó entre 2005 y 2008, mediante entrevistas con directivos y empleados de esta ONG así como con algunos productores. De igual manera tuvimos la oportunidad de participar en dos congresos internacionales de IFAT (Quito 2005, Blankenberge 2007), y revisamos literatura tanto sobre nuestro caso como sobre el movimiento internacional de comercio justo.

\section{UN CASO DE COMERCIO JUSTO EN ECUADOR}

En el sur de Quito se inició la conformación de Comunidades Eclesiales de Base, CEB, desde los 70. A mediados de los 80, estas CEB, inspiradas en la teología de la liberación, se organizaron en grupos en torno a la comercialización, y más tarde se constituyeron como ONG con el apoyo de un sacerdote. Este hecho daba vigencia jurídica al esfuerzo 
asociativo de comunidades y organizaciones principalmente urbanas articuladas con el objetivo de disminuir la cadena de intermediación en la comercialización de productos básicos de consumo masivo. De ahí, se puso en marcha un proceso de comercialización alternativo que consistía en la adquisición al por mayor de productos industrializados de primera necesidad — arroz, azúcar, sal, aceite, harinas, etc.—, y su distribución a un precio sin ánimo de lucro. El dispositivo introducido por ONG no apuntaba a la acumulación de capital $^{25}$ sin embargo se iniciaba un largo proceso de tensiones entre la lógica del «intercambio»y aquella del «don».

«MERCADITOS» O LA PREDOMINANCIA DEL «DON»

La primera comisión de trabajo de ONG se organizó alrededor de la decisión de conseguir productos a precios más convenientes. Se centralizaban las demandas hasta alcanzar volúmenes que la permitieran negociar en condiciones más ventajosas. ${ }^{26}$ La distribución de productos se llevaba a cabo con la participación activa de los miembros de las CEB. Ellos mismos recibían en la casa parroquial los productos, conciliaban las cuentas, y los entregaban a los coordinadores del sector para su venta y/o intercambio con otros productos, recuperando de esta manera una forma de trueque. Llegaron a existir más de 120 grupos de distribución a finales de los años ochenta. Luego de algunos meses de funcionamiento ONG tuvo pérdidas, dado que algunas familias no pagaron a tiempo sus deudas. Fue entonces cuando empezó a constituirse una estructura administrativa para responder de mejor manera a la comercialización. 
Se multiplicaron los partícipes tanto del lado de los oferentes de productos como de los demandantes. Llegaron campesinos de Lloa con papas, de Zumbahua con granos, de Esmeraldas con banano y otros productos tanto de la costa como de la sierra de Ecuador. Del lado de la demanda, ya no solo participaban los miembros de las comunidades eclesiales de base, sino cualquier morador del barrio donde ellas se organizaban. Más tarde ONG creció en diversas ciudades y pueblos del país. Esta etapa se denominó «mercaditos». Las ventas al inicio se realizaban en un día fijo según el barrio, pero luego se vio la necesidad de abrir tiendas barriales permanentes. De esta manera se multiplicaban los participantes y beneficiarios de las acciones, en una lógica espontánea y poco planificada. Y la administración se vio crecientemente desbordada por las nuevas relaciones y compromisos con nuevos actores. En esta etapa se realizaron asambleas mensuales de las organizaciones socias, a lo que se destinaban recursos importantes para financiar el desplazamiento mensual de diversos actores que reflexionaban sobre los avances de ONG y sus servicios.

Para ONG, en esta etapa, el «precio justo» ofrecido al consumidor era un precio más bajo que aquel tasado por los comerciantes al detalle. Se sacaba ventaja de las economías de escala en la compra de grandes cantidades de producto, que luego serían vendidas al detalle sin integrar en el costo final los costos de transacción. Este ahorro era posible gracias a una mano de obra voluntaria, así como gracias a la utilización de recursos logísticos «gratuitos» tales como el espacio físico de las parroquias y el trasporte subsidiado por la Iglesia o filántropos ligados a ella. En esta etapa, la «traducción» era 
innecesaria puesto que todos comprendían los enunciados iniciales en términos de solidaridad espontánea, beneficio mutuo y deber cristiano de apoyarse entre ellos.

DIVERSIFICACIÓN, EXPORTACIÓN Y TRADUCCIONES ENTRE MÚLTIPLES ACTORES Y RELACIONES

En 1988, la tasa anual de inflación en Ecuador se disparó a 85.71\%. ${ }^{27}$ La ONG no pudo ser ajena al proceso inflacionario. Eliminó los créditos, a finales de año presentó pérdidas importantes y algunas organizaciones socias se separaron de ella. Los directivos y empleados tenían concepciones distintas sobre la lectura de la crisis y sus soluciones, así como sobre la relación con la Iglesia católica. Se optó por conjugar una organización burocrática que administrara a $\mathrm{ONG}$, con rezagos de la estructura más horizontal del movimiento y participación de diversas organizaciones sociales en las reuniones anuales de asamblea. La mayor parte de costos fijos de ONG llegaron a financiarse con donaciones de la cooperación europea.

En este contexto, surge la idea de la exportación de productos con el propósito de diversificar mercados y ser menos vulnerables a las crisis internas del país. En 1990 se realizaron las primeras exportaciones a Italia. Se calcula que ese año salieron del país unos 40.000 dólares en artesanías, a pesar de las dificultades en los procesos administrativos y de producción que encontraron los grupos en su paso del mercado nacional al internacional. Los primeros compradores fueron organizaciones ligadas a la red de comercio justo en Europa: Cooperazione Terzo Mondo y Commercio Alternativo, adheridas a IFAT y EFTA Europe. Al mismo tiempo, ONG abrió el programa de exportación de cacao con ayuda de la 
cooperación internacional. En 1991, se adhiere a la organización internacional de comercio justo, IFAT, e ingresa en la red internacional. En 1993, ONG realizó la primera exportación de cacao de fino aroma hacia Europa, para Max Havelaar - comercio justo- y un comprador convencional en Holanda. Las organizaciones de base también comenzaron a pensar en alternativas de exportación de otros productos como café y maíz. Asimismo, ONG abrió un fondo de ahorro y crédito, el «fondo solidario», en el que se pagaba una tasa pasiva más alta que otras cooperativas y bancos privados, y que tenía el propósito final de asegurar la liquidez y disponibilidad de fondos para invertir en las nuevas iniciativas de exportación.

A este proceso de exportación e internacionalización le acompañó inevitablemente una lógica más centrada en la planificación y el cálculo, es decir, aquella lógica del intercambio mercantil, misma que en ocasiones tuvo que enfrentarse con la lógica centrada en la multiplicación de relaciones que continuaba alimentándose en ONG. La instalación de nuevas bodegas en nuevas ciudades, por ejemplo, era siempre precedida por reuniones o asambleas provinciales, con amplia participación de organizaciones de base y de segundo grado, por negociaciones, acuerdos y fiestas. Si bien en un inicio se había pensado que fueran las diversas organizaciones sociales, a través de una instancia representativa, las que condujeran ONG, en la práctica esta concepción fue reemplazándose por una estructura de organización burocrática, recurriendo a justificaciones tales como: los altos costos de los encuentros nacionales, ${ }^{28}$ la necesidad de contratar a personal profesional que cubriera el trabajo que se realizaba por voluntarios... 
Paralelamente, las actividades de las tiendas comunitarias así como en los mercaditos decayeron. En 1992 atravesaban algunos problemas que fueron resaltados por una evaluación interna: las organizaciones socias mostraban poco interés en los cursos de contabilidad y de salud; en la comercialización se observaba una descapitalización creciente, así como problemas de calidad de los productos, dificultades en el trasporte hacia las organizaciones socias, e informalidad en los procedimientos administrativos y de registro contable. En 1994 y 1995, ONG presentó pérdidas sucesivas en esta rama de actividad por el portafolio vencido de sus clientes. Las tiendas, que en ese momento eran sesenta, fueron paulatinamente cerradas así como las bodegas.

La concepción de «precio justo» poco a poco se va ligando a la calidad de los productos de exportación; a mayor calidad, mayor precio. Sin embargo, se mantienen con menor importancia las líneas de comercialización interna y de disminución de precios para sectores rurales y urbanos marginales. Los procesos de traducción entre productores ecuatorianos y compradores/consumidores internacionales devienen el corazón de las acciones de ONG. Es necesario traducir las demandas y características deseadas por los compradores para que los productores puedan responder de la manera más cercana y adecuada. Al mismo tiempo, es necesario traducir las expectativas y la situación de los productores en términos comprensibles para el movimiento internacional de comercio justo, con el propósito de «posicionarse» en el mercado internacional y lograr el crecimiento de las ventas. 


\section{DOLARIZACIÓN Y PREDOMINIO DEL «CÁLCULO»}

En enero de 2000 se decide implementar en Ecuador la dolarización. Esta decisión siguió a una crisis profunda del sistema financiero que se explicitó en 1999 y que significó la quiebra de la mayor parte de los bancos privados con actividades en el país. Como la mayor parte de ecuatorianos, ONG perdió parte de sus depósitos que se encontraban confiados a algún banco y tuvo serios problemas de liquidez. En este contexto, decide posicionarse fundamentalmente en el mercado externo. En 2001, ONG emprende un proceso de reestructuración interna mediante el cual separaba sus acciones en dos divisiones: por una parte, las unidades con fines comerciales que debían manejarse como empresas y presentar márgenes aceptables de rentabilidad, y por otra parte la unidad de intervención social dedicada a ejecutar proyectos con fondos de cooperación externa. Se procede a implementar una contabilidad analítica que pueda reflejar claramente los centros de costos y que permita identificar las actividades de pérdida.

Con el propósito de trasparentar los resultados de las actividades y acercarse al manejo racional, fundamentado en el cálculo del sector privado, ONG contrató profesionales de este sector. Se esperaba que las empresas operaran dentro de una lógica de eficiencia y rentabilidad de tal manera que pudieran generar el excedente necesario para sustentar el funcionamiento y los proyectos del área social. Estas definiciones además reducían la participación de las organizaciones sociales al encuentro en una asamblea anual. $^{29}$

Como parte de las nuevas estrategias y desafíos de mejoramiento de la calidad de los productos para el mercado internacional, ONG decide entrar en un proceso de 
certificación internacional. En un documento de la Cooperación Suiza para el Desarrollo se dice que ONG

... es una institución moderna en el sentido amplio del término; un ejemplo ilustrativo de ello es el haber alcanzado la Certificación de Calidad ISO 9001: 2000 y tenerla como estrategia fundamental para la perdurabilidad institucional; es una de las pocas fundaciones del país en haberlo logrado. El volumen anual de negocios [comercio más proyectos de financiamiento]... es de 18 millones de dólares. Trabaja en 16 Provincias, con 142 grupos organizados que corresponden a unos 5.000 beneficiarios.

Efectivamente ONG logró superar la crisis bancaria del país y el cambio de moneda al dólar, así como innumerables crisis internas, y también logró mejorar la calidad de los productos de exportación. Estos logros requirieron la introducción de cambios en los engranajes de la organización, mismos que se consideraron necesarios para sobrevivir al proceso nacional de dolarización y alcanzar una relativa estabilidad financiera. En 2006, la ONG, bastante flexible en términos de respuesta a nuevos contextos socioeconómicos, emprendió un nuevo proceso de reestructuración interna con el propósito de reducir costos a través de la racionalización y la organización por procesos. De esta manera, las diferentes líneas de comercialización pasan a compartir algunos procesos que hasta ese momento se habían especializado según el producto; nos referimos a procesos y costos, por ejemplo, de embalaje, bodegaje, márquetin. Asimismo se eliminan algunas líneas como aquella de comercialización de productos de primera necesidad, puesto que para esta época las redes de comercio convencional han ingresado con sus servicios a los barrios periféricos de las 
ciudades con costos que por el alto volumen de ventas pueden reducirse considerablemente. La ONG ha decidido orientarse más bien al trabajo con los productores campesinos, especialmente en productos de exportación como el cacao de fino aroma. En el contexto de canasta de productos, se observa que la artesanía y de mercado interno han perdido importancia relativa frente a productos como el cacao de exportación, que constituye el primer producto en ventas — casi 80\% de las ventas en 2007.

Estas decisiones, totalmente razonables y racionales, si se examinan bajo el prisma de los costos implican también una disciplina de cálculo que no solo compete a ONG sino también a los productores que trabajan con ella. Ligada además a la estrategia privilegiada de exportación, se encuentra la necesidad de mantener las cuentas al día con el Estado y esta se ha constituido en una línea prioritaria de capacitación de los beneficiarios: ayudarles primeramente a contar con las autorizaciones fiscales pertinentes para la comercialización de productos, y luego apoyarles en declarar periódicamente los ingresos provenientes de esa actividad. Pero asimismo este hecho es comprensible como fruto de los esfuerzos por apoyar a los productores para explotar ventajas comparativas en el mercado internacional, es decir, de apoyarlos a insertarse en el sistema y sobrevivir en él.

La ONG es una organización muy activa en el movimiento de comercio justo a escala internacional y forma parte de IFAT. En 2005, ONG logró que el congreso mundial del IFAT se reuniera en Quito, Ecuador. Asimismo ONG ha sido el eje de la organización regional de IFAT en América latina, misma que se denomina Red Latinoamericana de Comercialización Comunitaria, RELACC. 
El«precio justo» actualmente es el precio que ONG puede obtener en el mercado internacional sobre la base de la calidad del producto, misma que se mide a través de empresas de certificación internacional. La mayor parte de exportaciones se orientan actualmente al mercado convencional, puesto que la red internacional de comercio justo no tiene la capacidad de absorción de grandes cantidades de cacao. Con respecto a los productores, no todos ellos se encuentran ligados a ONG en esquemas de larga duración, y se observa un predominio porcentual de relaciones de mercado. Se realizan esfuerzos considerables para estandarizar la calidad del cacao y mantener la certificación internacional de calidad; se organiza la producción, la cosecha, poscosecha y bodegaje, esto se traduce en mayores precios pagados a los productores.

Los procesos de traducción, en este contexto, se dan en un solo sentido. Se trata de traducir las exigencias del mercado para que los productores comprendan lo que se debe hacer a la hora de obtener mayor calidad y, consecuentemente, precios más altos. Dado que la mayor parte de compradores son convencionales y que incluso en el movimiento de comercio justo las preocupaciones giran casi exclusivamente en torno a la calidad, las operaciones de traducción desde los productores hacia los compradores y consumidores han perdido importancia y sentido.

\section{CONCLUSIONES}

El movimiento de comercio justo surgió en la década de los 60 a raíz de la preocupación sobre los términos del intercambio entre los países desarrollados y aquellos que se 
encontraban «en desarrollo». Estas iniciativas que se plasmaron en declaraciones internacionales en el seno de Naciones Unidas y de la Iglesia católica tuvieron eco y se cristalizaron en acciones concretas en diversos países.

Ecuador es uno de estos países en los que una iniciativa ligada inicialmente a la Iglesia católica logra congregar la participación primeramente de barrios pobres de Quito para luego albergar a diversas organizaciones sociales de base alrededor de un proyecto de comercialización. Una revisión de la historia de esta organización constituye una ilustración de los logros y los obstáculos que ha enfrentado el movimiento de comercio justo en las tres últimas décadas. Su arranque estuvo marcado por el entusiasmo de diversos actores que hicieron proliferar el espíritu inicial del «don» en la organización. Mientras que las diversas crisis, no solo institucionales sino también nacionales e internacionales, y sobre todo la presión de la globalización y la dolarización del país, han conducido a sus actores a desplazarse poco a poco y a veces abruptamente hacia una lógica más centrada en el «intercambio» a través de la planificación, el cálculo y la búsqueda de una calidad estandarizada.

En el ámbito internacional, el movimiento de comercio alternativo trata también sobre temas como la necesidad de una estandarización de criterios para la construcción de una marca que pueda enfrentar la constante presión del mercado, la necesidad de implementar procesos de control de calidad, o la necesidad de una separación entre los productores y los intermediarios en el seno de la Organización Internacional de Comercio Justo, IFAT. Poco a poco las barreras que se habían forjado con respecto al comercio convencional, tanto en nuestro caso como en la esfera internacional, han ido 
desapareciendo en un afán por ampliar los mercados y llegar a consumidores que aun teniendo la conciencia de un comercio más justo requieren productos más estandarizados y que se ofrezcan en las grandes cadenas de distribución, pues su gestión del tiempo hace cada día más difícil la visita de comercios pequeños especializados.

Con nuestro caso hemos pretendido aportar a la discusión sobre el alcance y los límites que encuentran actualmente las estrategias de un comercio alternativo. 


\section{BIBLIOGRAFÍA}

Banco Central del Ecuador, 1989-2007, Boletín anual. Quito.

Ben-Ner, A. y T. Van Hoomissen, 1991, «Nonprofit organizations in the mixed economy: a demand and supply analysis». The nonprofit sector in the mixed economy. Annals of public and cooperative economics, vol. 62, núm. 4, pp. 519-550, oct.-dec., A. BenNer y B. Gui (eds.). CIRIEC, De Boeck-Wesmael, Belgium.

Bisaillon, V., 2005, «Le commerce équitable au Forum Social Mondial. Porto Alegre, 26 au 31 janvier». Bulletin Oeconomia Humana, vol. 3, núm. 2, pp. 5-7, mars.

Boltanski, L., 2000, El amor y la justicia como competencias: tres ensayos de sociología de la acción. Amorrortu editores, Buenos Aires.

Boltanski, L. y E. Chiapello, 1999, Le nouvel esprit du capitalisme. Editions Gallimard, Paris.

Callon, M., 1986, «Eléments pour une sociologie de la traduction. La domestication des coquilles Saint-Jaques et des marins pêcheurs en baie de Saint-Brieuc». L'Année sociologique, vol. 36, pp. 169-208. París.

1991, Réseaux technico-économiques et irréversibilités. Editions de 1'Ecole des Hautes Etudes en Sciences Sociales, Paris.

1999, «Essai sur la notion de cadrage-débordement». En Innovations et performances. Approches interdisciplinaires, pp. 399-431, D. Foray y J. Mairesse (eds.). Ecole des Hautes Etudes en Sciences Sociales, Paris. 
Callon, M. y B. Latour, 1997, «“Tu ne calculeras pas!”, ou comment symétriser le don et le capital”. Revue du MAUSS semestrielle, núm. 9, 1. ${ }^{\text {er }}$ semestre, pp. 45-70. La Découverte, Paris.

Castel, R., 1995, Les transformations de la question sociale. Chronique du salariat. Fayard, Paris.

Clastres, P., 1980, Recherches d'anthropologie politique. Editions du Seuil, Paris.

Defourny, J., L. Favreau y J.-L Laville, 1998, Insertion et nouvelle économie sociale. Un bilan international. Desclée de Brouwer, Paris.

Defourny, J. y J. L. Monzón Campos (eds.), 1992, Economie sociale: entre économie capitaliste et économie publique. Ed. De Boeck Université, Bruxelles.

Demoustier, D., 2001, L'économie sociale et solidaire. S'associer pour entreprendre autrement, pp. 114-115. Ed. La Découverte et Syros, Paris.

Espinosa, B., 2006, «Respeto, traducción y desarrollo». Ponencia presentada en el Encuentro de LASA, julio, mecanuscrito. Quito.

Fama, E. F. y M. C. Jensen, 1983, «Agency problems and residual claims». Journal of Law and Economics, vol. XXVI, núm. 2, pp. 327-349, june.

Favereau, O., 1989, «Marchés internes, marchés externes». Revue économique, vol. 40, núm. 2, pp. 273-328, mars.

FINE, 2001, Memento du commerce équitable. mimeo, Gand, Belgique. 
Gendron, C., 2005, «Deux commerces équitables? La voix du Sud au FSM 2005. Compterendu des ateliers sur le commerce équitable au Forum social mondial du Porto Alegre 2005». Oeconomia Humana, vol. 3, núm. 2, pp. 8-9. Chaire de responsabilité sociale et de développement durable. Université du Québec à Montréal, Québec.

Hansmann, H., 1980, «The role of nonprofit enterprise». Yale Law Journal, núm. 89, pp. 835-901.

Henaff, M., 2002, Le prix de la verité: le don, l'argent, la philosophie. Ed. Seuil, Paris.

Johnson, P., 2003, «Le commerce équitable, entre transformation et régulation socioéconomique». Oeconomia Humana, vol. 1, núm. 4, pp. 2-4. Université du Québec à Montréal, Québec.

Latour, B., 1991, Nous n'avons jamais été modernes. Essai d'anthropologie symétrique. Ed. La Découverte, Paris.

2005, Un monde pluriel mais commun. Entretiens avec François Ewald. Ed. De L'Aube, Paris.

Laurent, P.-J., 1998, Une association de développement en pays mossi: le don comme ruse. Ed. Karthala, Paris.

2005, Notes du cours «Anthropologie du Développement». Université Catholique de Louvain, Louvain-la-Neuve.

Mauss, M., 2004 [1950], Sociologie et anthropologie. Ed. PUF, Paris.

Nyssens, M., 1998, «Raisons d’être des associations et théorie économique». Discussion paper, núm. 9811. IRES, UCL, Belgium. 
Otero, A.-I., 2004, «Análisis y posicionamiento del comercio justo y sus estrategias: una revisión de la literatura». Les cahiers de la Chaire-collection recherche, núm. 042004, Chaire de responsabilité sociale et de développement durable. Université du Québec à Montréal, Québec.

Papa Paulo VI, 1967, Populorum Progressio. Lettre Encyclique de la Sainteté le Pape Paul VI sur le Développement des Peuples. En http://www.vatican.va. Vatican. (Fecha de consulta : abril 2007).

Provost, A.-C., 2002, Analyse de la coexistence d'organisations non lucratives, lucratives et publiques dans le secteur des maison de repos: une approche par les logiques de qualité. Université Catholique de Louvain, Faculté des sciences économiques, sociales et politiques, thèse, núm. 397. Louvain-La-Neuve.

Ricoeur, P., 2005, Sobre la traducción. Paidós, Buenos Aires.

Salamon, L. M., 1995, Partners in Public Service: Government-Nonprofit Relations in the Modern Welfare State. The Johns Hopkins University Press, Baltimore.

Sennett, R., 2003, Respect. De la dignité de l'homme dans un monde d'inégalité, p. 255. Ed. Hachette, Paris.

SIISE/INEC, 2001, Censo de Población y Vivienda, Versión 3.5. Quito.

UNCTAD, 1995, Documentos básicos. Naciones Unidas, Nueva York y Ginebra. En http://www.unctad.org (Fecha de consulta: abril de 2007).

Van Campenhoudt, L., 2001, Introduction à l'analyse des phénomènes sociaux. Ed. Dunod, Paris. 
Weisbrod, B. A., 1977, The Voluntary Nonprofit Sector. Ed. Heath, Lexington, Massachussetts.

Williamson, O. E., 1979, «Transactions-costs economics: the governance of contractual relations». Journal of Law and Economics, núm. 22, pp. 233-261.

Zaccaï-Reyners, N., 2006, «Respect, réciprocité et relations asymétriques». Esprit, consacré aux nouvelles figures du soin, núm. 321, janvier.

\section{Notas}

${ }^{1}$ Este documento recoge algunos avances de la investigación de tesis doctoral de la autora en la Universidad de Lovaina, Bélgica, cuyos promotores son R. Cobbaut y T. Périlleux.

${ }^{2}$ This document draws on some advances in the author's doctoral research at the University of Louvain, Belgium, whose promoters are R. Cobbaut and T. Périlleux.

${ }^{3}$ UNCTAD 1995.

${ }^{4}$ Papa Paulo VI 1967.

${ }^{5}$ P. Johnson (2003: 2).

${ }^{6}$ La sigla corresponde a su antigua denominación «International Federation for Alternative Trade».

${ }^{7}$ FINE (2001: 22).

${ }^{8}$ V. Bisaillon (2005: 5-7).

${ }^{9}$ C. Gendron (2005: 8-10).

${ }^{10}$ J. Defourny, L. Favreau, J.-L. Laville (1998: 11).

${ }^{11}$ Según Favereau (1989: 276), este tipo de teorías toman en cuenta las organizaciones como modo de asignación de recursos alternativo al mercado, aunque mantienen el postulado de racionalidad sustancial de las teorías estándar. La organización es aquí definida como conjunto de reglas y como entidad colectiva. Estos dos elementos sugieren una cooperación intencional que se opone a la cooperación no intencionada del mercado.

${ }^{12}$ No es un criterio de eficacia pero implica una reducción del riesgo de malversación de subsidios de las finalidades para las cuales fueron otorgados.

${ }_{13}^{13}$ A.-C. Provost 2002.

${ }^{14}$ Cfr. Defourny et al. 1998.

${ }^{15}$ Defourny et al. (1998: 35).

${ }^{16}$ D. Demoustier (2001: 114-115).

${ }^{17}$ R. Castel 1995.

${ }^{18}$ Otras respuestas con el mismo objetivo constituyen la generación de nuevas cooperativas que agrupan profesionales como los artistas para beneficiarse de economías de escala y defender su independencia, así como las asociaciones de consumidores. Cfr. D. Demoustier (2001: 90).

${ }^{19}$ P. Clastres (1980: 127-145).

${ }^{20}$ P. Clastres (1980: 130).

${ }^{21}$ Cfr. O. Favereau 1989.

${ }^{22}$ M. Callon y B. Latour (1997: 46).

${ }^{23}$ N. Zaccaï-Reyners (2006: 95). 
${ }^{24}$ Citado por P. Ricoeur, 2005, Sobre la traducción. Paidós, Buenos Aires.

${ }^{25}$ Siguiendo a Boltanski y E. Chiapello (1999), de las diferentes caracterizaciones del capitalismo o de los capitalismos retendremos una fórmula mínima que hace hincapié en la exigencia de acumulación ilimitada de capital mediante medios formalmente pacíficos. La perpetua puesta en circulación del capital dentro del circuito económico con el objetivo de extraer beneficios, es decir, de incrementar el capital que será a su vez reinvertido de nuevo, sería lo que caracterizaría primordialmente al capitalismo y lo que le conferiría esa dinámica y esa fuerza de trasformación que han fascinado a sus observadores, incluso a los más hostiles. La acumulación de capital no consiste en un acaparamiento de riquezas, es decir, de objetos deseados por su valor de uso, su función ostentatoria o como signos de poder. Las formas concretas de la riqueza —inmobiliaria, bienes de equipo, mercancías, moneda, etc.— no tienen interés en sí y pueden suponer incluso debido a su falta de liquidez un obstáculo para el único objetivo realmente importante: la trasformación permanente del capital ... en producción, la producción en dinero y el dinero en nuevas inversiones.

${ }^{26}$ La primera compra consistió en 100 quintales de arroz y 100 de azúcar, los mismos que se guardaban en un local de la casa parroquial.

${ }^{27}$ Banco Central del Ecuador, boletín anual 1989.

${ }^{28}$ La movilización y hospedaje de los representantes de las organizaciones a los encuentros nacionales eran financiados por ONG con fondos provenientes de donaciones.

${ }^{29}$ El modelo de planificación participativa preveía otras instancias y mecanismos de participación más directos.

Fecha de recepción: 05 de mayo de 2008.

Fecha de aceptación: 03 de octubre 2008. 